Revue d'histoire de l'Amérique française

QV REVUE D.HISTOIRE DE L'AMÉRIQUE FRANÇAISE

\title{
Le métier d'infirmière : savoirs féminins et reconnaissance professionnelle
}

\section{Yolande Cohen et Michèle Dagenais}

Volume 41, numéro 2, automne 1987

URI : https://id.erudit.org/iderudit/304549ar

DOI : https://doi.org/10.7202/304549ar

Aller au sommaire du numéro

\section{Éditeur(s)}

Institut d'histoire de l'Amérique française

\section{ISSN}

0035-2357 (imprimé)

1492-1383 (numérique)

Découvrir la revue

\section{Citer cet article}

Cohen, Y. \& Dagenais, M. (1987). Le métier d'infirmière : savoirs féminins et reconnaissance professionnelle. Revue d'histoire de l'Amérique française, 41(2), 155-177. https://doi.org/10.7202/304549ar
Résumé de l'article

Cet article porte sur le processus de professionnalisation des infirmières au Québec, au début du $20^{\mathrm{e}}$ siècle. En remontant aux origines de la profession, il s'agit d'étudier comment des infirmières, sur la base du savoir féminin " care " -, ont procédé pour établir leur métier puis en faire une profession, et inscrire leur rôle dans le vaste champ de la santé. C'est notamment par le biais des associations professionnelles, que les infirmières vont réussir à transformer leur participation dans ce domaine, au départ associée au travail bénévole et charitable, en travail professionnel salarié.

Deux hôpitaux montréalais - l'Hôpital général de Montréal et l'Hôpital Ste-Justine - permettront de suivre les étapes de ce processus qui mène à la création de l'Association des gardes-malades enregistrées, en 1920. Seront mises en parallèle les stratégies utilisées par différents réseaux de femmes, anglophones et francophones, pour valoriser des pratiques et des savoirs reliés au soin et à l'entretien du corps. Les unes, de l'Hôpital général de Montréal, s'appuient sur un ensemble de savoirs spécifiques pour affirmer leur compétence propre, tandis que les autres, de l'Hôpital Ste-Justine, ont recours à la communauté de femmes pour obtenir la reconnaissance du rôle nouveau des femmes dans l'organisation de la santé publique. Chaque groupe réalise toutefois l'importance de fonder sa démarche sur l'amélioration de l'éducation et sur une plus grande formalisation des soins infirmiers. C'est pourquoi, les infirmières se doteront rapidement d'associations autonomes, en vue d'établir les critères et le standard d'accès à la profession auprès des instances publiques.
Tous droits réservés @ Institut d'histoire de l'Amérique française, 1987

Ce document est protégé par la loi sur le droit d'auteur. L'utilisation des services d'Érudit (y compris la reproduction) est assujettie à sa politique d'utilisation que vous pouvez consulter en ligne.

https://apropos.erudit.org/fr/usagers/politique-dutilisation/ 


\title{
LE MÉTIER D'INFIRMIÈRE: SAVOIRS FÉMININS ET RECONNAISSANCE PROFESSIONNELLE ${ }^{1}$
}

\author{
YOLANDE COHEN \\ MICHËLE DAGENAIS \\ Département d'histoire \\ Université du Québec à Montréal
}

\begin{abstract}
RÉSUMÉ
Cet article porte sur le processus de professionnalisation des infirmières au Québec, au début du 20e siècle. En remontant aux origines de la profession, il s'agit d'étudier comment des infirmières, sur la base du savoir féminin - «care» - , ont procédé pour établir leur métier puis en faire une profession, et inscrire leur rôle dans le vaste champ de la santé. C'est notamment par le biais des associations professionnelles, que les infirmières vont réussir à transformer leur participation dans ce domaine, au départ associée au travail bénévole et charitable, en travail professionnel salarié.

Deux hôpitaux montréalais - l'Hôpital général de Montréal et l'Hôpital Ste-Justine - permettront de suivre les étapes de ce processus qui mène à la création de l'Association des gardesmalades enregistrées, en 1920. Seront mises en parallèle les stratégies utilisées par différents réseaux de femmes, anglophones et francophones, pour valoriser des pratiques et des savoirs reliés au soin et à l'entretien du corps. Les unes, de l'Hôpital général de Montréal, s'appuient sur un ensemble de savoirs spécifiques pour affirmer leur compétence propre, tandis que les autres, de l'Hôpital Ste-Justine, ont recours à la communauté de femmes pour obtenir la reconnaissance du rôle nouveau des femmes dans l'organisation de la santé publique. Chaque groupe réalise toutefois l'importance de fonder sa démarche sur l'amélioration de l'éducation et sur une plus grande formalisation des soins infirmiers. C'est pourquoi, les infirmières se doteront rapidement d'associations autonomes, en vue d'établir les critères et le standard d'accès à la profession auprès des instances publiques.
\end{abstract}

\section{ABSTRACT}

This paper deals with the process of professionalization of nursing in Quebec at the beginning of the twentieth century. By examining the beginnings of the profession, it seeks to show how nurses used their female "caring» skills to characterize their occupation, turn it into a profession and thus define their place in the health care professions. Through professional associations, nurses changed their role from that of a benevolent and charitable endeavour to that of professional work for wages.

Two Montreal hospitals - the Montreal General Hospital and Ste. Justine Hospital - are the focus of the article. The steps leading to the formation of the Association of Registered Nurses, in 1920, are retraced. The paper examines the different strategies used by the francophone and

i Une partie de cet article a fait l'objet d'une communication présentée au Congrès annuel de la Société historique du Canada, en juin 1987. Il s'inscrit dans une recherche plus vaste que dirige Yolande Cohen, avec notamment Michèle Dagenais comme assistante de recherche, sur l'histoire comparée des métiers féminins. Nous tenons à remercier le Conseil de recherches en sciences humaines du Canada qui l'appuie financièrement, de même que les deux évaluateurs anonymes de la Revue d' histoire de l'Amérique française pour leurs judicieuses remarques. Nous voudrions finalement remercier Luc Lépine, archiviste aux Archives historiques de l'Hôpital SteJustine qui a grandement facilité nos recherches. 
anglophone women's networks in promoting the recognition of their caring skills and practices. Nurses from the Montreal General Hospital use skills derived from their experience to affirm their specific competence, while those from Ste. Justine Hospital rely upon the women's community to gain recognition of women's new role in the organization of public health care. Each group, however, is aware of the need for improved education and for a greater formalization of nursing practices. Thus nurses quickly set up autonomous associations, designed to define professional norms and criteria for the use of public bodies.

L'étude de la professionnalisation des infirmières au Québec révèle des stratégies complexes utilisées par différents réseaux et regroupements de femmes pour faire reconnaître et valoriser des savoirs reliés au soin et à l'entretien du corps. Étroitement dépendante des recherches effectuées sur l'histoire du travail féminin et de sa professionnalisation, l'histoire des infirmières traduit les aléas d'une carrière établie sur l'identification au sexe. La double utilisation qui est faite du savoir féminin - «care» - et de sa transmission particulière par des femmes exclusivement, fait de cette occupation l'un des premiers métiers féminins à vocation professionnelle.

Marquée par les nombreux travaux qui, ces dernières années, ont cherché à qualifier ce type d'emploi et à mesurer sa valeur dans la grille des professions, l'histoire des infirmières reste caractérisée par des analyses qui privilégient avant tout la discrimination sexuelle pour expliquer un statut qui oscille entre une semi-profession ${ }^{2}$, une occupation, et une profession ${ }^{3}$. Mais en même temps, ce champ d'investigation est lui-même l'objet de renouvellements importants ${ }^{4}$, et c'est un des objectifs de ce texte que d'y contribuer.

C'est en Angleterre que la célèbre Florence Nightingale ouvre la brèche par sa conception révolutionnaire du métier. Bien qu'elle considère les femmes comme des infirmières potentielles, toutes ne peuvent le devenir. Certes le sexe fonde le métier, et bientôt la profession, mais à certaines conditions. À la science des médecins, affirme Nightingale, doit s'ajouter le soin des malades; à la médication et aux traitements, la propreté, l'air frais, une attention suivie et soutenue aux malades. Le soin moral du patient est présenté comme étant aussi important que le soin du corps pour obtenir la guérison et même prévenir la maladie. Or

\footnotetext{
2 Amitai Etzioni, The Semi-Professions and their Organization: Teachers, Nurses, SocialWorkers (New York, Free Press, 1969).

3 Au Québec, de nombreuses recherches restent en deçà des efforts déployés ailleurs pour renouveler nos approches. Elles se sont appuyées sur la division sexuelle du travail entre médecins et infirmières - cure et care - pour aborder la question sous l'angle d'une discrimination sexuelle aboutissant à une dévalorisation du travail infirmier et au ghetto d'emploi féminin. Voir les études de L.-H. Trottier, Évolution de la profession infirmière au Québec de 1920 à 1980, mémoire de maîtrise (Sociologie), Université de Montréal, 1982; D. Gaucher, Division sexuelle du travail hospitalier et discriminations salariales, mémoire de maîtrise (Sociologie), Université de Montréal, 1982.

4 Eliot Freidson, Professional Powers. Study of the Institutionalization of Formal Knowledge (Chicago, University of Chicago Press, 1986).
} 
toutes ces qualités d'entretien sont inhérentes à la nature féminine. Toutefois, cette tâche de prendre également soin des âmes ne saurait être assumée par n'importe quelle femme. Seules celles faisant preuve d'une moralité irréprochable, possédant de grandes vertus, seront admises dans la profession et pourront se voir accorder le droit d'exercer une tâche aussi noble. Seule l'élite des femmes, en quelque sorte, pourra y être admise.

Qui dit élite dit formation et sélection. En qualifiant la fonction d'infirmière, Nightingale et, à sa suite, ses adeptes du nursing américain, établissent une distinction nette entre le sens commun des femmes, nécessaire au métier quoiqu'insuffisant, et les savoirs infirmiers. Elles ouvrent un nouveau champ de compétences basé sur la connaissance et l'expertise, le care ou l'entretien des malades, qui déterminera l'accès au métier et deviendra, plus tard, le fondement de la profession. On se demandera si cette distinction n'est pas à la source de l'ambiguité d'un métier où la hiérarchie et la compétition côtoient l'humilité de la vocation. Il reste que cette même ambiguïté permet aussi une percée inédite des femmes dans un champ dominé par les hommes, celui de la santé.

Au Québec, la profession apparaît comme le prolongement des mouvements de charité et de philanthropie, actifs pendant la seconde moitié du $19 \mathrm{e}$ siècle. A ce titre, les influences internationales, anglaise et américaine surtout, sont marquantes. Elles se traduisent par la nette conscience qu'ont les Canadiennes anglaises de faire oeuvre de pionnières au Canada tandis que, du côté francophone, le domaine de la charité et des soins continue d'être l'apanage des communautés religieuses. Cette double détermination s'entremêle curieusement au tournant du siècle pour donner naissance à l'idéologie sanitaire qui deviendra le leitmotiv de l'affirmation professionnelle des infirmières.

Deux hôpitaux montréalais d'inégale grandeur, l'Hôpital général de Montréal (Montreal General Hospital) et l'Hôpital Ste-Justine, nous permettront de suivre les étapes de cette histoire. Elle se solde par la création de l'Association des gardes-malades enregistrées de la province de Québec qui obtient le monopole de l'enregistrement en 1920. Le premier hôpital, anglophone et laïque, institue la première école d'infirmières au Québec. Il fait partie de la catégorie des grands hôpitaux canadiens qui seront à l'origine de la standardisation du fonctionnement hospitalier. Ce mouvement, importé des États-Unis et calqué sur le fonctionnement des grandes organisations sanitaires, se développe au début des années 1920. De moyenne importance, le second hôpital, francophone et également laïque, est fondé par un groupe de dames bienfaisantes de la société montréalaise et se spécialise dans le soin des enfants et de leurs mères. Bien qu'il fasse appel à la communauté des Filles de la Sagesse pour sa gestion interne, l'hôpital fonctionnera sous la direction d'un exécutif laïque. 
Ces deux études de cas nous permettront d'examiner deux types de stratégies utilisées par les infirmières pour faire reconnaître leur profession. Notre objectif est ici limité à l'histoire de ce groupe, laquelle se déroule dans des contextes bien particuliers. Jusqu'à la fin des années 1920 , en effet, les administrations hospitalières ont des fonctionnements très spécifiques qu'il ne s'agit pas de comparer. Mais parce que cette période est celle tumultueuse de l'élaboration des soins hospitaliers, elle permet de mieux comprendre comment se développe la nouvelle organisation du travail. Les infirmières vont alors tenter d'asseoir leur profession et d'établir une répartition du travail qui leur convient. Les premières, à l'Hôpital général de Montréal, font appel à un corps de savoirs spécifiques pour affirmer leurs compétences propres, tandis que celles de Ste-Justine ont recours à la communauté des femmes pour faire reconnaître le rôle nouveau des infirmières dans l'organisation de la santé publique. Chaque groupe comprend néanmoins la nécessité de fonder sa démarche sur ce qui deviendra une conception commune du métier: ce sera, d'une part, l'amélioration de l'éducation et la plus grande formalisation des soins infirmiers dont l'idéologie «sanitaire» leur fournit le contenu et, d'autre part, la construction d'associations autonomes qui négocieront auprès des instances politiques les critères et le standard d'accès à la profession. Dans cette démarche, aussi bien le code d'éthique définissant la responsabilité professionnelle que les structures hiérarchiques de la profession seront empruntés au fonctionnement des hôpitaux, modelé en grande partie par les médecins, sous la tutelle desquels les infirmières devront se placer. A ce niveau, il nous faudra évaluer clairement les alternatives qui se présentaient à elles quand elles ont choisi le domaine de la santé publique pour exercer leurs fonctions; car, de toute évidence, la domination de ce champ par une profession à haut prestige, établie sur des savoirs très formalisés, ne laissait qu'une place subordonnée à tous ceux qui n'avaient ni son autorité ni son statut ${ }^{5}$.

\section{LA POUSSÉE VERS LES SAVOIRS FÉMININS A L'HÔPITAL GÉNÉRAL DE MONTREAL}

Le cas de l'Hôpital général de Montréal est particulièrement révélateur des principaux enjeux qui conduisent les infirmières à faire reconnaître leur profession; on peut y voir une sorte de synthèse des expériences anglaise et américaine. Fondé en 1818 par un groupe de femmes bénévoles, la Female Benevolent Society, l'Hôpital général est une modeste institution de quatre lits. L'année suivante, c'est un groupe d'hommes d'affaires qui l'administre; par la suite, la gestion sera assu-

5 G. Dussault aborde bien la question de l'antériorité historique dans le champ occupationnel et souligne combien elle détermine souvent la façon dont les compétences sont distribuées et le monopole des premiers arrivés, sauvegardé; G. Dussault, «L'évolution du professionalisme au Québec», Relations indusrielles/Industrial Relations, 33,3: 429-460. 
rée grâce aux dons privés et aux octrois gouvernementaux, à l'instar des autres hôpitaux ${ }^{6}$. Se développant rapidement, l'hôpital doit avoir très tôt recours à une main-d'oeuvre importante pour assurer son bon fonctionnement. C'est là que la première école de gardes-malades du Québec est fondée en 1890. Tant sa création que la formation qu'elle dispense font de cette école un modèle et un exemple qui seront déterminants dans le développement de la profession au Québec.

L'ouverture d'une école de nursing à l'Hôpital général survient à l'issue d'une longue période de transition qui s'échelonne sur près de vingt ans ${ }^{7}$. La question des services d'une équipe de gardes-malades avait été éludée à cause des nombreuses oppositions qu'elle soulevait, dûes surtout au manque de moyens financiers. Jusque-là, l'entretien des malades était confié à des femmes mariées. Ces matrons, comme on les appelait à l'époque, étaient recrutées un peu partout: parmi les anciennes patientes de l'hôpital, les employées des cuisines ou encore les femmes de ménage. Elles devaient surveiller les dortoirs des patients, veiller au bon fonctionnement du ménage et à l'entretien des malades. Ces matrons étaient secondées dans ces tâches par des aides gardesmalades recrutées sensiblement de la même manière. Les soins proprement dits des malades étaient en général confiés aux étudiants en médecine qui faisaient leur internat à l'Hôpital général de Montréal ${ }^{8}$. Ainsi, on distingue déjà plus ou moins clairement les soins des malades de leur entretien.

Notons alors que ce n'est pas le besoin de main-d'oeuvre qui milite en faveur de l'école, mais plutôt une certaine conception des soins. Du côté des médecins, tous ne sont vraiment pas convaincus de la nécessité d'organiser une école de formation pour gardes-malades. Pour sa part, l'hôpital est en faveur de l'instauration d'un système de nursing permanent et plus fonctionnel. Les progrès de la médecine, la fréquentation croissante des hôpitaux par la population poussent l'administration hospitalière naissante à mieux organiser les soins infirmiers. L'emploi de gardes-malades qualifiées pourrait favoriser une meilleure implantation de l'hôpital dans la communauté. Ainsi, l'idée de leur formation est dans l'air et les plus avant-gardistes des médecins vont s'appuyer

6 H. E. MacDermott, History of the School of Nursing of the Montreal General Hospital (Montréal, The Alumnae Association, 1960), 1-3. Cet ouvrage, relativement bien connu, a permis de compléter et recouper, dans certains cas, les informations recueillies lors de nos recherches dans les archives de l'Hôpital général de Montréal.

7 Le premier projet de constitution d'une école remonte au milieu des années 1870; époque qui marque le début d'une série de tentatives en ce sens et qui n'aboutira qu'en 1890. Archives de l'Université McGill (AUMG), RG 96, Committee of Management of the Montreal General Hospital, Minutes, vol. C.21 1871-1875, 23 février 1874.

8 Ibid., 7-8. 
sur l'exemple britannique, déjà appliqué en Ontario, pour faire valoir leur demande'.

Dès 1874, l'administration de l'Hôpital général de Montréal s'adresse à l'école de Florence Nightingale pour obtenir l'envoi d'une garde-malade graduée chargée d'organiser son école de formation. $\mathrm{La}$ surintendante du St. Thomas Hospital accepte l'offre d'emploi, mais y adjoint un certain nombre de conditions, dont l'amélioration de l'hygiène à l'hôpital. Il faudra attendre un an avant que le projet ne se réalise; les conditions matérielles et sanitaires de cette institution sont loin d'être équivalentes à celles qui existent dans les hôpitaux anglais. L'Hôpital général de Montréal est beaucoup moins «moderne»: les malades sont entassés dans des chambres exiguës où manquent lumière et aération ${ }^{10}$. Malgré les efforts déployés par Miss Machin, la surintendante anglaise, c'est l'impasse entre la direction de l'hôpital et cette dernière, deux ans à peine après son arrivée. On lui reproche d'être trop extravagante et sans doute trop élitiste aussi, et de ne pas avoir réussi à former de nouvelles gardes-malades ${ }^{11}$.

Dès lors, et durant les dix années qui suivent, c'est le surintendant médical qui se chargera de la formation des gardes-malades, laquelle se résume le plus souvent à quelques démonstrations pratiques ${ }^{12}$. Et l'hôpital retourne à son ancien système de matrons pour assurer l'encadrement des gardes et le service de nursing. Au cours de cette période, bien des médecins se plaindront du nombre insuffisant d'infirmières et parfois même de la qualité des recrues. La difficulté du travail et la précarité des conditions de vie - manque d'espace adéquat pour les loger - provoquent un roulement important des gardes-malades et empêchent leur recrutement plus important et d'un niveau plus élevé. On assiste alors à une situation paradoxale où pour devenir infirmière diplômée, les candidates canadiennes doivent s'expatrier aux ÉtatsUnis $^{13}$.

9 Dans l'ensemble du Canada, seule l'Ontario est alors dotée d'une école de gardes-malades. L'ouverture d'une école dans cette province, au General and Marine Hospital de St. Catherines, en 1874 , intervient à l'issue d'une série de tentatives infructueuses entreprises depuis 1864. D. M. Jensen, History and Trends of Professional Nursing (St. Louis, The C. V. Mosby Co., 1950), 515-516.

${ }_{10}$ Une longue correspondance est entreprise entre Miss Machin, l'infirmière anglaise, et le comité d'administration de l'hôpital à ce sujet. AUMG, loc.cit., 2 février 1874 au 28 avril 1875.

11 Comme en fait état alors le rapport du comité financier de l'hôpital: «The management of the Hospital can be made more effective and economical by the... introduction of a Matron of the best experience... who should be placed over the nurses and women servants, who should be responsible for the cleanliness of the Hospital...» AUMG, loc.cit., vol. C.23 1876-1879, 24 septembre 1877 .

${ }_{12}$ AUMG, loc.cit., vol. C.24, 1879-1883, 31 juillet 1881.

13 Tel que le mentionne le rapport trimestriel de l'hôpital: «The nurses cannot be had for there is no room to accomodate them... If we had good rooms for the nurses I have not doubt many of the best young women of this country who find their way to American Hospitals would stay at home for their training and thus the standard of nursing in this country would be much arised.» AUMG, loc.cit., vol. C.26 1886-1889, 31 juillet 1887. 


\section{Miss Livingston et la création de la première école de formation de gardes-malades au Québec}

Sous la pression combinée des médecins, et au nom du progrès de la science et du développement de l'hôpital, le comité d'administration se décide enfin à reconnaître que l'embauche d'une infirmière dûment formée et diplômée est nécessaire. Cette fois, on se tourne vers les États-Unis pour recruter une diplômée d'expérience, la lady superintendant que beaucoup réclament.

C'est la désormais célèbre Gertrude E. N. Livingston qui obtiendra le poste ${ }^{14}$. Célèbre parce que lui reviendra le mérite d'avoir fondé la première école d'infirmières au Québec et une des premières au Canada $^{15}$, célèbre aussi car elle a contribué à rehausser le niveau de la profession, tâche à laquelle elle consacrera sa vie. Durant les vingt premières années de son mandat, elle devra se confronter aux questions complexes de la professionnalisation des infirmières. Elle réussira plus facilement que ses prédécesseurs à imposer sa manière de faire et de concevoir la profession ${ }^{16}$. Une de ses premières prérogatives sera de séparer les soins de l'entretien des malades. Elle-même aura la responsabilité exclusive de tout ce qui a trait au recrutement, à la formation et à la direction des gardes-malades, tandis que tout le domaine de l'entretien ménager sera sous la responsabilité d'une autre personne. Elle obtient également la permission d'être secondée par deux infirmières diplômées de son choix. Elle établit ainsi une distinction nette entre les gardes-malades et les autres femmes, membres du personnel hospitalier, qui justifie leur traitement particulier.

\section{La formation d' une élite}

L'école ouvre ses portes le premier avril 1890, quelques mois après l'arrivée de la surintendante ${ }^{17}$ et reçoit, au cours des deux premières années, près de 200 demandes d'admission ${ }^{18}$ annuellement. Peu pas-

14 Au moment où elle accepte l'offre de l'Hôpital général de Montréal, Miss Livingston ne possède qu'un simple diplôme de garde-malade. Elle occupait depuis peu un poste de surintendante des infirmières au New York Hospital. Elle restera à l'hôpital général de Montréal jusqu'à sa retraite en 1919. M. D. Jensen, op. cit., 515.

15 À la même période, le mouvement de professionnalisation des infirmières se développe partout à travers les États-Unis. Au cours des vingt dernières années du $19 \mathrm{e}$ siècle, le nombre d'écoles dans ce pays passe de 15 en 1880 à plus de 400 au début de 1900 , et le nombre de gardesmalades diplômées se multiplie tout aussi rapidement passant de quelque 150 infirmières à plus de 3000 . Voir à ce sujet l'ouvrage de B. Melosh, The «Physician's Hand». Work Culture and Conflict in American Nursing (Philadelphia, Temple University Press, 1982), en particulier le chapitre 2, «Hospital Schools of Nursing».

16 AUMG, loc.cit., vol. C.27 1889-1892, 24 et 30 janvier 1890.

17 L'hôpital compte alors 165 lits et il y a entre 30 et 35 gardes-malades non-diplômées en service. AUMG, loc.cit, vol. C.27 1889-1892, 4 février 1890.

18 Une première estimation du profil socio-démographique des candidates indique que plus de $50 \%$ des premières infirmières travaillaient déjà dans un autre domaine, au moment de leur demande d'admission. Cela donne à penser qu'elles entrevoyaient ce métier comme offrant des possibilités d'avancement social. L'analyse exhaustive des cheminements de carrière de ces filles est en cours. 
sent le test et seulement le sixième (soit 33 élèves au cours de la seconde année) termine le cours. Opérant une sélection très rigoureuse, Miss Livingston n'accepte que les meilleures postulantes. L'enjeu est de taille: il s'agit de se démarquer de l'image de la nurse ancienne manière, aux moeurs approximatives et sans qualification précise, et d'établir des critères élevés d'accès à la profession.

Le contenu de la formation reste encore sommaire, bien que plus formalisé. Le cours se résume pour l'essentiel à un apprentissage pratique, d'une durée de deux ans. Une première période de deux mois, dite de «probation», permet de sélectionner les candidates les plus aptes à le suivre. Les tâches de l'élève consistent à faire les lits, servir les repas, aider les patients à faire leur toilette... Par la suite, l'élève apprend à distribuer les médicaments aux malades, à s'enquérir de l'état général du patient: prendre la température, le pouls, le rythme de la respiration, bref à établir un premier diagnostic. Au cours de la seconde année, l'étudiante a la charge d'un dortoir et veille à transmettre aux nouvelles arrivantes les premiers rudiments de la profession. A l'issue de ces deux ans, les élèves passent une série d'examens sous la supervision d'un médecin et d'un chirurgien de l'hôpital, avant d'être reçues infirmières.

Dès le départ, la collation des diplômes revêt un caractère très solennel. En présence des médecins de l'hôpital ou de leurs représentants, de certains gouverneurs, d'amis et parents des futures gardesmalades, ces dernières doivent prononcer un serment professionnel qui les engage à faire honneur à la voie qu'elles ont choisie. Ce serment constitue un des éléments fondamentaux qui confirme le statut professionnel des infirmières en établissant leur responsabilité à l'égard des malades, mais aussi leurs devoirs envers la corporation.

Ainsi, avec l'arrivée de Miss Livingston, est désormais reconnue la nécessité de former des soignantes qui deviendront, après leurs cours, des infirmières. Si jusque-là l'élaboration du contenu de la formation et de sa durée a été quasi exclusivement le fait de la surintendante des gardes-malades, à partir du début du $20 \mathrm{e}$ siècle, tant le comité d'administration que le bureau médical expriment le désir d'intervenir. En ce sens, le tournant du siècle inaugure une nouvelle période. En 1899, le comité d'administration fait appel à la surintendante et au bureau médical dans le but d'élaborer un programme de formation plus systématique, qui tienne compte de la durée de chaque stage dans les différents départements de l'hôpital et de l'ordre de leur déroulement, au cours des trois années. Des cours théoriques, sur des sujets traitant d'anatomie, de chirurgie, de gynécologie et de diététique notamment, sont donnés le soir à $19 \mathrm{~h} 15$ ainsi que le jeudi après-midi et le samedi matin. L'enseignement est partagé entre la surintendante secondée par deux infirmières graduées et le bureau médical ${ }^{19}$. Quelques années plus

19 AUMG, loc.cit., vol. C.30 1898-1901, 30 janvier 1899 au 5 juin 1899. Voir également H. E. MacDermot, op. cit., 53. 
tard, en 1906, l'hôpital embauchera même une institutrice officielle pour l'école ${ }^{20}$. Cette répartition progressive des tâches conduira à l'intervention plus directe du corps médical dans l'élaboration du contenu de la formation. La perspective d'une formation plus complète, davantage axée sur l'acquisition de connaissances scientifiques, se profile clairement, donnant à l'école un statut plus prestigieux, et au métier ses fondements professionnels.

\section{La négociation d'un statut}

Pour l'hôpital, l'école de formation d'infirmières possède le double avantage d'attirer un nombre important de candidates et d'élèves susceptibles de fournir une main-d'oeuvre adéquate pour effectuer les tâches reliées au soin des malades, mais aussi de donner un statut et une visibilité à l'hôpital. C'est pourquoi le comité d'administration se garde d'interférer dans la formation des gardes-malades, qu'il délègue au bureau médical et à la surintendante. Là où il commence à y avoir problème, c'est quand les infirmières diplômées, engagées en plus grand nombre, cherchent à négocier, à titre professionnel, leur statut dans l'hôpital.

Dès le départ, l'embauche des gardes-malades salariées est l'objet de négociations serrées entre la direction de l'hôpital et la directrice des soins infirmiers. Les médecins sont réticents à leur reconnaître un statut de professionnelles. Une reconnaissance formelle et explicite équivaudrait à accepter une première division du champ médical ${ }^{21}$ et à partager, même si c'est de façon modeste, le pouvoir alors exclusif que possèdent les médecins dans ce domaine. Ne pouvant en évaluer toutes les conséquences, on préfèrera alors limiter le développement d'une classe de soignantes professionnelles. Jusqu'à la fin de la Première Guerre mondiale, les diplômées représenteront toujours moins de $10 \%$ du corps infirmier $^{22}$.

C'est pour faire face à ces limitations que les infirmières tentent différentes interventions en vue d'établir, dans les faits, la spécificité

20 Miss Shaw est une autre figure marquante de la profession. Graduée de l'Hôpital général de Montréal en 1896, elle occupe d'abord un poste du surintendante dans un petit hôpital à Boston. Elle obtient un diplôme en enseignement du nursing en 1903 du Teachers' College, rattaché à l'Université de Columbia, à l'issue de trois années d'étude. Pour plus de détails, consulter l'ouvrage de Barbara T. Logan, In Caps and Gowns. The Story of the School for Graduate Nurses McGill University, 1920-1964 (Montréal, McGill University Press, 1966), 33-35.

${ }_{21}$ A cette époque, en effet, la division du travail dans le secteur hospitalier est très sommaire. Les médecins et les infirmières constituent les deux intervenants les plus importants. Comme l'utilisation des nouvelles technologies médicales (rayons-x p.e.) est encore restreinte, il faut attendre le tournant des années 1920 avant que ne se développe le groupe des employés «paramédicaux».

${ }_{22}$ Ainsi en est-il en 1893, par exemple, alors qu'il n'y a que trois graduées sur un total de 50 gardes-malades, comme en 1905 , où l'on retrouve seulement six professionnelles diplômées sur un total de 78 élèves infirmières. 
et l'exclusivité de leur formation. Dans un premier temps, elles voudront occuper différents postes dans l'hôpital et ainsi utiliser leur compétence exclusive pour combler les besoins nouveaux. Au milieu des années 1890, par exemple, Miss Livingston obtient l'accord du Comité exécutif pour qu'une garde-malade soit affectée exclusivement à la salle d'opération. Cette dernière aura alors pour tâches de préparer tous les instruments nécessaires à ce chapitre, procéder à leur stérilisation et leur rangement. Dans la même veine, une infirmière sera nommée responsable du département de la scarlatine. Au cours de cette première étape, les tentatives en vue de formaliser les compétences infirmières ne s'appuient donc pas encore sur une technicité, comme ce sera le cas dans les décennies suivantes. Les infirmières cherchent alors davantage à étendre leur présence à tous les niveaux du travail hospitalier.

La question de la rémunération des soins infirmiers traduit bien la volonté des infirmières de se faire entendre. Recevant jusque-là des indemnités, elles chercheront à obtenir des salaires. Mais, au départ, dès que le conseil d'administration consent à offrir un salaire régulier à une garde-malade graduée, il s'empresse aussitôt de préciser qu'il ne l'accorde que de manière exceptionnelle ${ }^{23}$. Ce n'est qu'en 1905 que l'hôpital accepte d'établir une échelle de salaires pour le personnel infirmier, en fonction du degré de responsabilité ${ }^{24}$. Dès lors, on reconnaît ipso facto la spécificité et surtout la valeur de leur travail.

La première manche en vue de la reconnaissance de leur fonction propre est ainsi obtenue par les infirmières, du moins à l'Hôpital général de Montréal. Leur salaire se paye au prix d'une élitisation de leur statut: ce qui ne porte pas à grande conséquence tant qu'elles sont peu nombreuses. Dans une deuxième période, la négociation empruntera le cheminement plus classique de l'association. A travers elle, commence la lutte pour la règlementation de la profession. Cependant, avant d'aborder cette seconde étape, voyons comment à la même époque, mais dans un tout autre milieu, l'Hôpital Ste-Justine, les infirmières envisagent les soins infirmiers et la profession.

\footnotetext{
23 Comme une résolution de la direction l'expose clairement ici: «... the engagement of the 'Day nurse in charge' was to be for one year only. The Secretary to write to Miss Livingston that the expectation of the Committee of Management is that, in the future, the Training School will furnish from its graduates, some possessing the requisite qualifications for the position of Nurses in charge». AUMG, loc. cit., vol. C.27, 1889-1892, 29 février 1892.

24 Il y a alors cinq gardes-malades graduées à l'emploi de l'hôpital, qui sont rémunérées comme suit: une infirmière responsable de nuit qui gagne $55 \$$, une de jour à $45 \$$, une infirmière assistante surintendante reçoit $40 \$$, une infirmière à la salle d'opération touche $30 \$$ et celle qui est en charge du département de gynécologie, 25\$ par mois. AUMG, loc. cit., vol. C.32, 1904-1907, 27 mars 1905.
} 


\section{DÉVOUEMENT FÉMININ ET DIVISION DU TRAVAIL A L'HÔPITAL STE-JUSTINE}

Contrairement aux vieux hôpitaux qui, comme l'Hôtel-Dieu, ont le plus souvent été conçus, organisés et gérés par des communautés religieuses, l'Hôpital Ste-Justine est créé au début du siècle par un groupe de «bienfaitrices laïques». Le docteur Irma Levasseur, première femme voulant combler l'inadéquation des soins donnés aux enfants, fait campagne pour la création d'un hôpital qui leur serait spécialement destiné. Mais ne jouissant ni du capital ni des appuis nécessaires à une telle entreprise, elle se voit contrainte d'abandonner son projet, qu'elle remet néanmoins entre les mains d'une dame respectable, Madame Alfred Thibodeau. Le 30 novembre 1907, cette dernière réunit dans son salon ses amies, dames influentes et bien placées, qui s'enthousiasment pour le projet et font campagne pour le réaliser ${ }^{25}$.

Très vite, Justine Lacoste de Gaspé Beaubien prend la direction de l'entreprise. Une loi constitue en corporation les fondatrices et leurs successeurs, tandis que, le premier janvier 1908, le premier bureau médical est formé. Aussitôt, soit le 12 février suivant, ce dernier décide de créer une école de formation pour gardes-malades ${ }^{26}$. Plusieurs comités sont alors chargés par l'exécutif et le bureau médical de s'assurer de la collecte de fonds et du fonctionnement de l'hôpital: comités de publication, de souscription, de lingerie, et de l'économie interne.

D'emblée l'hôpital se présente comme la contribution originale et spécifique des femmes en vue de l'amélioration de la santé des enfants (lutte contre la mortalité infantile), du développement de l'hygiène et des soins aux mères. Grâce à leur place privilégiée dans la société canadienne-française, ce petit groupe de femmes obtient rapidement, mais pas toujours facilement, la possibilité de développer un projet qui leur est propre et qu'elles entendent bien contrôler:

Grâce à ce puissant patronage..., nous sortîmes victorieuses d'une petite lutte, engagée entre nous, les femmes qui voulions la plus grande liberté pour travailler le plus efficacement possible à notre chère oeuvre et messieurs les hommes, qui jaloux de leurs droits ne voulaient pas, sans se faire prier un peu, les partager avec nous. ${ }^{27}$

25 Madame Louis de Gaspé Beaubien, née Justine Lacoste et fille de Sir Alexandre Lacoste, conseiller législatif, ancien président du Sénat canadien et juge en chef à la Cour d'appel du Canada; Madame Théodule Bruneau, née Lucie Lamoureux dont le mari est médecin en chef de l'Hôtel-Dieu; Mlle Euphrasine Rolland, fille de l'honorable sénateur J.-Damien Rolland et Madame Arthur Berthiaume, née Blanche Bourgoin, épouse du président du journal La Presse. Voir R.-S. Lasalle, Madame Louis de Gaspé Beaubien, sa vie, son oeuvre, 1907-1967 (Hôpital Ste-Justine, s.d.), 5-6. 12 février 1908.

27 AHSJ, Rapport de la secrétaire du Comité exécutif, Premier rapport annuel, 1908. 
Armées d'une volonté inébranlable, elles établissent le fonctionnement de l'hôpital sur une semblable conception: mélange complexe de missionnariat et de bénévolat, alimenté tant par la charité chrétienne que par le développement de la médecine, qu'elles veulent mettre au service des mères et des petits malades. Elles sont convaincues qu'elles réussiront à faire marcher l'hôpital en mobilisant leurs compagnes et leurs maris ou amis médecins. Rapidement, toutefois, elles réalisent qu'il leur faut s'organiser.

\section{Les soins aux malades: un apostolat}

Au début, cependant, et pour combler les besoins de l'hôpital, un consensus est facile à obtenir sur la nécessité de rassembler tous les efforts pour le faire fonctionner. En janvier 1908, une garde-malade diplômée est engagée, Mlle Léda Larue, au salaire annuel de $240 \$^{28}$. Elle sera entourée de jeunes filles, élèves-infirmières et «aides-maternelles», à qui on offre une indemnité de $5 \$$ et $3 \$$ par mois respectivement, en plus du gîte et du couvert. Cette modeste rémunération se justifie par le fait que l'hôpital compte leur donner en échange de leurs services une formation qui mène à une carrière. Mais le contenu de la formation, alors dispensée par les médecins, n'est que très vaguement défini ${ }^{29}$. Dans ces conditions, l'hôpital éprouve très tôt de sérieuses difficultés à s'adjoindre du personnel hospitalier, même peu qualifié. Malgré les nombreuses tentatives faites par sa directrice, Madame Beaubien, pour alléger la durée de la formation des gardes-malades (à deux ans seulement au lieu de trois) afin d'attirer plus de vocations, et en ouvrant un cours par semaine au public pour trouver les aides maternelles nécessaires, les résultats ne sont guère probants. En fait, dès la fondation de l'école des gardes-malades ${ }^{30}$, cette répartition du travail montre ses failles. D'abord il n'est pas simple de recruter du personnel spécialisé sans le payer; ensuite le concours des médecins tant à l'enseignement qu'aux soins ne peut être obtenu sans être réglementé.

Les jeunes filles recrutées ${ }^{31}$, au départ, auprès des curés de campagne ou même dans les écoles ménagères ${ }^{32}$, ne sont pas légion. Ainsi

${ }^{28}$ Ce salaire représente le quart de celui touché par la surintendante des infirmières de l'Hôpital général de Montréal, à la même époque.

29 Des médecins, désignés par le Bureau médical, doivent donner des conférences sur des sujets telles l'anatomie, la diététique, la pathologie interne, les maladies de l'enfance, mais aucune modalité n'est précisée sur la manière dont se dérouleront les cours; AHSJ, Bureau médical, Procès-verbaux 1908-1928, 11 mars 1908.

31 L'école ouvre officiellement le premier décembre 1909; AHSJ, Rapport annuel, 1910. phique, qui nous permettra d'identifier avec précision qui sont les élèves infirmières, n'est pas complétée.

${ }_{32}$ Pour faciliter le recrutement de jeunes filles, le comité d'administration propose: «... (d')envoyer à Messieurs les curés à la campagne surtout, les prospectus des aides-maternelles et de leur demander à engager les jeunes filles de leur paroisse obligées de pourvoir à leur existence, à embrasser cette profession si avantageuse». AHSJ, Comité exécutif, Procès verbaux, vol. 1, 1907-1908, 28 septembre 1908. 
en 1911 par exemple, il n'y a que six gardes-malades d'inscrites. De même en 1914, soit plus de cinq ans après la création de l'école, l'hôpital ne compte que quinze élèves gardes-malades ${ }^{33}$. Ces dernières se heurtent d'emblée à cette façon de concevoir l'hôpital comme devant être une communauté de femmes unies pour assurer le bien-être et la santé des petits, lesquels doivent passer avant toute autre considération, monétaire, professionnelle ou autre.

\section{L'appel aux religieuses: les Filles de la Sagesse}

La directrice de Ste-Justine fait finalement appel à Mgr Bruchési, alors archevêque de Montréal, qui lui suggère d'engager une communauté religieuse pour la seconder et assurer le fonctionnement interne de l'hôpital (cuisine, buanderie et soins infirmiers). Quand, le premier mars 1910, le conseil d'administration signe avec les Filles de la Sagesse ${ }^{34}$ un traité leur confiant la charge de la gestion interne de l'hôpital en échange du gîte, du couvert et d'une certaine formation, la direction accepte théoriquement de partager certaines de ses prérogatives avec la Supérieure de la communauté et, notamment, celle de la responsabilité des soins infirmiers. De fait, en optant pour une solution qu'elle pensait plus économique et plus conforme à ses idéaux, la directrice élude le problème posé par le besoin d'une main-d'oeuvre qualifiée. La communauté éprouvera des difficultés à s'implanter dans un milieu où l'ensemble des décisions revient à l'exécutif de l'hôpital, et à Madame Beaubien en particulier, et en ressentira une impression de mauvais traitement ${ }^{35}$; mais, surtout, la délicate question de la répartition des tâches entre les gardes-malades et les religieuses sera à l'origine de nombreux conflits. L'épineuse question des tâches domestiques, partie intégrante de la fonction des religieuses, se pose de nouveau lorsque les gardes-malades refusent de les accomplir. Elle signifie une soumission des religieuses aux laïques, ce qui, dans le contexte montréalais, reste assez exceptionnel. Ailleurs, à l'Hôtel-Dieu par exemple, les religieuses, qui possèdent et dirigent l'institution, exigent des gardes d'accomplir une bonne partie des menus travaux.

33 Jusqu'au milieu des années 1920 , le nombre d'élèves demeure peu important. Ainsi, il n'est que de 25 en 1920, mais augmente sensiblement par la suite puisque l'on dénombre 57 infirmières en formation en 1926 et 104 en 1928. AHSJ, Rapports annuels, 1914-1928.

${ }_{34}$ Peu connue, cette communauté, originaire de Poitiers (France), établit ses premières oeuvres dans le diocèse d'Ottawa, un orphelinat en 1884, puis des écoles paroissiales à SaintJovite en 1890. Les institutions hospitalières dont elle se chargera ( 5 au total) se consacrent au soin des enfants malades. On compte 56 religieuses Filles de la Sagesse dans le diocèse de Montréal au milieu des années 1920. J.-P. Archambault, Sur les pas de Marie. Congrégations de femmes au Canada français (Montréal, Imprimerie du Messager, 1929), 283-285.

35 Ce qui fait aussi l'objet d'une volonté d'ajustement de la part de la Supérieure. AHSJ, dossier 27, Filles de la Sagesse 1909-1947, correspondance entre Madame Beaubien et la Supérieure de la communauté, mars 1913. 
La question de l'accomplissement des tâches domestiques à l'Hôpital Ste-Justine illustre de façon cruciale les difficultés qu'il y a à circonscrire les attributions des gardes-malades, mais surtout à délimiter ce qui relève du soin, de l'entretien du corps et de l'amélioration du bien-être des patients. Toutes les fonctions sont incluses dans le terme générique de soins infirmiers (care), que toute femme dévouée devrait pouvoir accomplir sans grande difficulté. Pourtant la valeur attribuée aux différentes tâches est déjà clairement définie. Le développement des savoirs infirmiers a déjà engendré une distinction nette et définitive entre les soins et les savoirs féminins, relégués au simple sens commun. C'est pourquoi, derrière la revendication des religieuses de ne plus avoir à porter seules le fardeau de l'entretien domestique de l'hôpital, se profile leur anxiété d'être exclues du système de formation et d'accès au métier de garde-malades.

\section{Soins infirmiers et infirmières bénévoles}

La dimension presque familiale de l'hôpital à ses débuts et la mission d'apostolat que sa directrice a imprimée aux soins infirmiers conduisent à des heurts et réajustements dans les règlements et le fonctionnement de l'école d'infirmières. Contrairement à l'Hôpital général de Montréal, il n'y a pas, au départ, de garde-malade diplômée religieuse ou laïque - - qui dispense la formation. Hormis les fonctions hiérarchisées des sept religieuses qui occupent les postes de supérieure, d'infirmière en chef, de pharmacienne (qui est en même temps chargée de l'école des gardes-malades) et quatre infirmières, les six autres gardesmalades laïques occupent indistinctement ${ }^{36}$ les mêmes fonctions, qu'elles soient en cours d'apprentissage (élèves-infirmières) ou déjà formées. Ce qui conduira plusieurs d'entre elles à démissionner très peu de temps après leur engagement ${ }^{37}$. Ces débuts chaotiques ne sont guères tempérés par le règlement draconien qui régit l'activité quotidienne des gardes-malades, religieuses comprises, et par des conditions de travail pénibles ${ }^{38}$.

Après 1922, on assistera à une lente reconnaissance du travail des infirmières ${ }^{39}$ quand une spécialisation accrue et un plus haut degré de

36 AHSJ, Rapport annuel, 1911.

37 . C'est le cas de Mlle Léda Larue, infirmière diplômée de l'Hôpital Notre-Dame, qui donne sa démission en 1909, quelques mois avant la fin de son contrat d'une durée d'un an, à titre de surintendante à l'Hôpital Ste-Justine. Citons également celui de Mlle Lamoureux qui ne demeure à ce poste que deux mois, à la suite de Mlle Larue. AHSJ, dossier 20-1, Infirmières en service: Correspondance.

${ }_{38}$ A cette époque tant la vie durant les heures de service que celle à l'extérieur fait l'objet d'une surveillance étroite, tout comme dans les autres hôpitaux d'ailleurs. Les gardes n'ont alors droit qu'à une heure de repos par jour (pour un service de 12 heures) et un après-midi de congé par semaine. Elles peuvent également sortir le soir mais doivent être de retour à $21 \mathrm{~h} 30$. AHSJ, dossier 25-N, Nursing (1924-1958), «Règlements des gardes-malades», vers 1910.

39 Cette reconnaissance intervient dans un tout autre contexte et résulte notamment des pressions qui seront faites à l'endroit de l'hôpital par l'Association des gardes-malades enregistrées de la province de Québec. Voir la dernière partie du présent article à ce sujet. 
technicité viendront diversifier les fonctions qu'elles occupent. Cependant jusque-là, l'Hôpital Ste-Justine ne considère guère les soins infirmiers comme étant spécialisés, mais plutôt comme une extension de l'activité bénévole et charitable des femmes. D'ailleurs, un service d'infirmières bénévoles spécialement recrutées et formées sur le tas devient la caractéristique particulière qui distingue Ste-Justine. Car non content d'avoir recours au bénévolat, l'hôpital organise et assoit un système de soins parallèle à celui des infirmières. Au fur et à mesure qu'il se développe et se spécialise, ce corps d'infirmières bénévoles exerce une pression considérable sur l'engagement d'infirmières salariées, contestant même leurs prérogatives sur le terrain de la formation, puisqu'un certificat leur sera également décerné.

Ainsi donc, les savoirs infirmiers comme les cours de formation restent flous et indifférenciés, malgré l'école, à cause d'une politique de recrutement et d'expansion de l'hôpital basée sur l'apostolat des femmes. Le rêve de Madame Beaubien serait en voie de se réaliser: une communauté de femmes unies et dévouées autour d'une mission hygiéniste participe de plein pied à l'amélioration des soins prodigués à une population qui en a un urgent besoin. Mais le développement des connaissances médicales et de la conscience plus sanitaire que charitable de certains groupes d'infirmières de Montréal orientent ce projet sur une tout autre voie. La pression de certains médecins, qui veulent élever le niveau de qualification des gardes-malades, et celle des associations d'infirmières, extérieures à Ste-Justine, donnent la priorité à des considérations plus strictement professionnelles que bénévoles et charitables.

\section{CLUBS, ASSOCIATIONS ET CORPORATION: LE CHEMINEMENT DES INFIRMIERES VERS LE STATUT PROFESSIONNEL}

Ces deux exemples illustrent clairement comment les infirmières cherchent, timidement à Ste-Justine, de façon plus explicite à l'Hôpital général de Montréal, à établir leurs prérogatives dans les querelles qui les opposent, individuellement d'abord, puis collectivement, aux administrations hospitalières. Très vite, elles refusent d'effectuer des tâches ménagères qui, selon elles, ne relèvent pas de leur compétence. Ensuite, elles demandent l'octroi de postes de responsabilité dans les différents services de l'hôpital. Puis, c'est autour de la rémunération et des conditions de travail que les plaintes et représentations surgissent. Peu nombreuses et non organisées, les infirmières n'en ressentent pas moins la nécessité de faire valoir leur contribution aux soins des malades. Limitées par le pouvoir des médecins et celui des directions hospitalières, elles choisissent de se regrouper entre elles pour tenter de se délimiter un champ de pratique et de l'instituer. Divers regroupements, d'abord axés sur le secours mutuel et la sociabilité, les conduisent à se 
poser le problème de leur avenir. A l'instar des autres pays, elles réalisent que le seul moyen de se protéger est de contrôler entièrement l'accès à la profession, donc la formation, le registre et la collation des grades. Prenant appui sur les autres corporations professionnelles, elles découvrent les vertus de l'association professionnelle.

Les influences internationales sont très présentes dans la constitution même d'un mouvement associatif chez les infirmières. Le premier regroupement d'infirmières canadiennes se fait en liaison avec les Américaines. En 1893 est formée The Association of Superintendants of Training Schools for Nurses of the United States and Canada, dont le but est de: «...establishing and maintaining a universal standard of training, and by promoting fellowship among its members by meetings...» ${ }^{40}$. Quelques années plus tard, en 1896, une seconde association sera mise sur pied regroupant cette fois les infirmières graduées, réunies autour d'associations de diplômées ${ }^{41}$.

Au Canada et au Québec, les préoccupations reliées au contrôle de la profession émergent dans le contexte de son développement rapide, à partir du tournant du siècle, avec la création d'écoles de gardes-malades dans de nombreux hôpitaux ${ }^{42}$. Les associations d'infirmières diplômées d'hôpitaux vont jouer un rôle de premier plan en vue d'obtenir la reconnaissance de la profession. Ce n'est pas tant par des activités revendicatrices qu'elles se déploient, mais plutôt par la promotion d'une idéologie professionnelle et de l'esprit d'appartenance à un corps, qu'elles insufflent à leurs membres.

\section{Le club des infirmières: "The Alumnae Association of the Montreal General Hospital Training School for Nurses»}

A l'Hôpital général de Montréal, dès le début du 20e siècle, certaines actions sont entreprises de façon isolée, en vue de protéger la profession naissante. Il importe d'abord de protéger les emplois et donc d'établir des critères pour privilégier les membres d'une même école, d'un même hôpital, d'une même catégorie ${ }^{43}$. C'est dans ce contexte

\footnotetext{
40 Margaret M. Allemang, Nursing Education in the United States and Canada, 1873-1950. Leading Figures, Forces, Views on Education, thèse de Ph.D. (Education), University of Washington, 1974, 70 .

41 Il s'agit de l'Associated Alumni of the United States and Canada; ibid., 88.

42 À Montréal seulement, cinq hôpitaux fondent leur propre école de formation pour gardesmalades laïques. Il s'agit de l'Hôpital Royal Victoria (1893), de l'Homeopathic Hospital (1895), du Women's Hospital (autour de 1900), de l'Hôpital Notre-Dame (1899) et de l'Hôtel-Dieu (1901). Archives de l'Ordre des infirmières et des infirmiers du Québec (AOIIQ), boîte 10, Écoles d' infirmières.

43 À la demande expresse de Miss Livingston, la surintendante des infirmières, l'hôpital accepte d'adopter un règlement favorisant exclusivement ses infirmières diplômées. C'est ainsi que, dorénavant, les médecins travaillant en pratique privée ne devront embaucher, pour les seconder, que les gardes-malades de cet hôpital, au détriment de celles formées dans d'autres hôpitaux. AUMG, RG 96, Committee of Management, Minutes, vol. C.31, 1901-1904, 6 avril 1903.
} 
qu'émerge l'idée de former une association; il s'agit de se doter d'une porte-parole des diplômées pour négocier, en lieu et place des membres dispersées, les ententes pour défendre leur statut et, si nécessaire, pour améliorer leur formation.

Les infirmières diplômées de l'hôpital décident donc de créer leur propre association en 1905. Elles sont neuf gardes-malades, incluant la surintendante, à l'origine de la constitution de l'association qui prend le nom évocateur de Livingston Club. Dès le départ le but que se fixe la nouvelle association est clair: «... (to) set a high standard of professional work subject to rules and regulations...»4. Au cours des deux premières années de son existence, le regroupement des infirmières graduées fait davantage office de club social, de lieu d'échanges, de réseau d'information et de sociabilité. Ses activités consistent à recevoir des conférenciers qui abordent différents aspects de la professsion, et à faire salon de thé $e^{45}$. Des rencontres mensuelles se déroulent au domicile d'une des membres. L'assistance aux réunions est assidue, - elle totalise dès le départ près d'une trentaine de membres - bien que restreinte et sélective. Sur quelque 220 graduées de l'Hôpital général de Montréal en 1904, l'Association regroupe 73 membres dès la fin de 1905.

A partir de 1907, l'Association s'engage résolument dans des activités du type secours mutuel pour ses membres, plus inspirées par la promotion de la profession. Sa première réalisation importante est la mise sur pied d'un registre pour les infirmières graduées en service privé, en $1907^{46}$. Le registre fonctionnera de façon autonome et rapidement, on assure, avec les cotisations, un poste rémunéré à une infirmière chargée de sa tenue. A la même époque, l'association opte pour un nouveau nom plus conforme à sa vocation et devient le Montreal General Hospital Alumnae Association ${ }^{47}$.

Des liens étroits unissent l'Association des infirmières de l'Hôpital général aux autres regroupements canadiens ${ }^{48}$. Elles s'entendent pour demander en priorité le pouvoir d'accorder l'enregistrement aux infir-

44 D'ailleurs les fondatrices entendent bien se doter des moyens nécessaires pour le faire respecter: «... and nurses not living up to the standard prescribed will be compelled to resign...» AUMG, RG 96, Minutes of the Alumnae Association of the Montreal General Hospital, C.421, item 2/1/1, 17 avril 1905.

45 AUMG, loc. cit., 17 avril au 30 mai 1905.

46 AUMG, loc. cit., 10 avril 1907.

47 Elle change une nouvelle fois de nom en 1909 et adopte définitivement celui de: Alumnae Association of the Montreal General Hospital Training School for Nurses. AUMG, loc. cit., 13 janvier 1909.

48 En particulier avec l'Association des infirmières canadiennes, mise sur pied en 1908 par la réunion de toutes les associations d'infirmières diplômées canadiennes existantes et la branche canadienne de l'Association des surintendantes d'hôpitaux. Reproupant 16 associations, elle est alors formée dans le but d'être représentée à la prochaine réunion du Conseil international des infirmières qui doit se tenir à Londres en 1909. Pour plus de détails, voir l'ouvrage de J. M. Gibbon et M. S. Mathewson, Three Centuries of Canadian Nursing (Toronto, MacMillan of Canada, 1947), 357 ss. 
mières diplômées. Devant la diversité des formations des gardes-malades et la prolifération de pratiquantes non-diplômées, seule une loi, obligeant les infirmières à s'enregistrer, donnerait aux dirigeantes du nursing une source centralisée de pouvoir, qui leur permettrait de réglementer la profession. Du début des années 1910 au début des années 1920 , chaque association provinciale tentera d'obtenir, avec plus ou moins de succès, ce privilège ${ }^{49}$.

\section{La bataille de l'enregistrement}

$\mathrm{Au}$ Québec, cette question est inscrite à l'agenda des infirmières diplômées et plus particulièrement de celles de l'Hôpital général de Montréal. Après de nombreuses tractations entre les différents groupes, une organisation provinciale est constituée. En 1917, les infirmières anglophones, de Montréal essentiellement, forment The Graduate Nurses Association of the Province of Quebec, dans le but d'obtenir l'enregistrement provincial des gardes-malades. Deux ans seulement après sa fondation, l'association regroupe 660 membres, réunies dans diverses associations d'infirmières diplômées ${ }^{50}$. Elle obtient facilement, en 1920, l'adoption d'une loi qui accorde le privilège du titre réservé, et adopte le nom d'Association des gardes-malades enregistrées de la province de Québec/Association of registered Nurses of the Province of Quebec (AGMEPQ).

Cette loi ne manquera pas de soulever des oppositions passionnées, particulièrement dans le milieu francophone. Dans un premier temps, elle passe, cependant, sans trop de remous. Un certain laxisme du système juridique à l'égard des professions ${ }^{51}$, ainsi que le rôle éminent joué par les infirmières pendant la Première Guerre mondiale, ont créé un climat favorable à leur projet. S'appuyant sur une conception de la profession qui l'établit sur des critères similaires aux autres domaines de la santé, plutôt que sur les méthodes empiriques, et adoptant un code d'éthique et de responsabilité, l'AGMEPQ a su neutraliser ses opposants les plus farouches et s'allier une classe importante de

49 En 1922, les neuf provinces canadiennes sont dotées d'une loi conférant, aux associations provinciales respectives, le privilège de donner le titre d'infirmière enregistrée à leurs membres. Notons qu'il n'est alors pas obligatoire de porter ce titre pour pouvoir exercer la profession, bien qu'il confère à celles qui le possèdent un prestige certain. Voir l'ouvrage de $\mathrm{J}$. M. Gibbon et $\mathrm{M}$. S. Mathewson, op. cit.

50 Frances E. Upton, 25 ans de collaboration (1920-1945) (Association des gardes-malades enregistrées de la province de Québec, 1946), 4-5.

51 Gilles Dussault souligne la facilité avec laquelle les lois octroyant un titre réservé et le monopole de la pratique étaient passées, à cette époque. Il suffisait qu'un groupe suffisamment structuré en fasse la demande, avec l'appui d'un député, pour que la loi soit adoptée; Gilles Dussault, loc. cit, 439 ss. 
médecins ${ }^{52}$. Surtout, l'AGMEPQ n'empiétait pas sur les compétences des médecins et tâchait de délimiter le rôle des gardes-malades aux seuls soins infirmiers, effectués en complément et sous la direction des médecins.

Aussi l'AGMEPQ obtient-elle le monopole de l'enregistrement qui lui permet d'établir clairement ses objectifs. Il s'agit:

...(de) créer un corps de gardes-malades possédant une formation et une compétence complètes pour soigner les malades, de procurer à celles qui possèdent cette formation et cette compétence les moyens de se faire reconnaitre du public ainsi que favoriser l'efficacité et le bien être des gardes-malades en général. ${ }^{53}$

La loi stipule que pour devenir membre de l'association et porter le titre de «garde-malade enregistrée», il faut être âgée d'au moins 23 ans, de bonne réputation, avoir obtenu un certificat d'une école de formation rattachée à un hôpital d'une capacité d'au moins 50 lits, et donnant un cours «d'enseignement hospitalier systématique» d'au moins trois ans. Les candidates, pour être admises dans l'association, devront en outre passer un examen contrôlé par elle. Celles qui pratiquent déjà ou qui suivent un cours de formation au moment de l'adoption de la loi sont admises comme membres, sans examen, pourvu qu'elles remplissent les autres conditions prévues par la loi.

S'inscrivant dans le courant favorisant la modernisation des soins et le développement des installations hospitalières, l'AGMEPQ acquiert assez rapidement un statut et un prestige, si bien que les différents hôpitaux vont chercher à ce que leurs écoles de gardes-malades soient reconnues par cette dernière. Mais, en même temps, les autres clauses contenues dans la loi vont soulever un tollé de protestations de la part des petits hôpitaux de moins de 50 lits ou qui n'offrent pas une formation conforme aux exigences de la loi. La première opposition à la loi provient de 65 gardes-malades diplômées de petits hôpitaux francophones (la Miséricorde, Ste-Justine et Dupont). Elles demandent que les exigences d'admission soient assouplies pour les inclure. Dans un deuxième temps, un groupe de médecins rattachés à l'Université de Montréal, ou pratiquant dans de petits hôpitaux, s'opposera également à la loi de 1920 et tentera d'obtenir le contrôle de plusieurs aspects touchant la profession. Une série d'amendements seront apportés au texte de loi original.

\footnotetext{
52 Ceux d'entre eux qui comptaient développer avec les infirmières la pratique de la santé publique. Ces derniers privilégient une conception semblable à la même époque, dans le but d'étendre le champ de leur pratique à la médecine préventive et à l'hygiène publique qui se développe de façon importante au Québec; M. Fournier et al., Sciences et médecines au Québec, perspectives socio-historiques (Québec, Institut québécois de recherche sur la culture, 1987), 9-19.

53 Statuts du Québec, 10 Georges V, chapitre 141.
} 
La version amendée de la loi ${ }^{54}$, adoptée en mars 1922, qui octroie à l'Université de Montréal la certification des infirmières, constituera un recul certain pour l'AGMEPQ. Les exigences d'admission sont d'abord assouplies; l'âge minimum est abaissé à 21 ans et les gardesmalades pratiquantes qui n'ont suivi qu'un cours de deux ans, antérieurement au présent amendement, pourront désormais être admises sans autre formalité. Pour inclure les francophones dans ses rangs, l'AGMEPQ se voit donc obligée d'accepter des gardes-malades qui ont un niveau de formation inférieur à ses exigences premières. Le second amendement stipule que: «...les gardes-malades diplômées par une université de la province après avoir suivi un cours d'au moins trois ans dans une école reconnue par la faculté de médecine de cette université (Université de Montréal) sont inscrites sans autre examen.» ${ }^{55} \mathrm{Par}$ cet autre amendement, l'AGMEPQ perd donc le monopole ou si l'on préfère l'exclusivité de la collation des diplômes, puisque l'université a la possibilité de faire admettre, sans examen, les élèves formées dans les hôpitaux qu'elle reconnaît.

\section{L'Hôpital Ste-Justine et l'enregistrement des infirmières}

La principale source d'opposition à l'origine de ces amendements provient d'un groupe d'infirmières, dont plusieurs diplômées de l'Hôpital Ste-Justine. À la différence des principaux hôpitaux de Montréal qui se sont dotés d'associations d'infirmières autonomes, Ste-Justine n'en possède pas. Les statuts et règlements qui régissent leur travail et leur vie à l'hôpital sont très semblables à ceux négociés par les Filles de la Sagesse; et les infirmières bénévoles n'ont guère d'intérêt professionnel à faire valoir. Le problème de l'enregistrement ne se posera réellement que pour une infime catégorie de gardes-malades tandis que la valeur du diplôme décerné par l'école reste entière. Or, c'est précisément cette question qui sera soulevée par le monopole de l'enregistrement, octroyé par la loi de 1920 à l'AGMEPQ. Dès lors qu'une association nationale de gardes-malades s'arroge le droit de contrôler l'accès à la profession, le système-maison mis en place par l'Hôpital Ste-Justine s'avère caduc. L'AGMEPQ est une instance perçue comme faisant la promotion d'idéaux de professionnalisme opposés au corporatisme pratiqué à Ste-Justine et qui plus est, animée par ce qui semble être un groupe de Canadiennes anglaises protestantes.

Une telle alliance ne peut donc se réaliser sans heurts et c'est «naturellement» que Madame Beaubien fera appel aux médecins pour contrebalancer le pouvoir, qu'elle juge exhorbitant, accordé par la loi à l'AGMEPQ. Il s'agira de faire valider, par la Faculté de médecine de l'Université de Montréal, les cours reçus par les infirmières à Ste-Jus-

54 Statuts du Québec, 12 Georges V, chapitre 131.
Ibid., article 8b. 
tine. Les détails qui entourent cette procédure sont forts significatifs puisque c'est au nom du développement du métier d'infirmière chez les Canadiennes françaises catholiques, que l'argumentation est menée ${ }^{56}$. Par un tel biais, on oblige l'AGMEPQ à reconnaitre et donc admettre sans qu'elle puisse le contrôler, l'accès à la profession des gardesmalades formées ailleurs mais munies du diplôme de l'Université de Montréal.

Ainsi, les médecins sont réintroduits par l'Hôpital Ste-Justine dans un débat à l'écart duquel l'AGMEPQ avait voulu les tenir. Cette dernière avait pris bien soin de délimiter clairement les prérogatives des infirmières de celles des médecins, en établissant ses critères sur des savoirs complémentaires à ceux des médecins et qui, bien que sous leur tutelle, échappaient largement à leur contrôle, tant en ce qui avait trait à leur gestion qu'à leur développement.

Certes, on peut se demander, comme Barbara Melosh le fait ${ }^{57}$, si l'orientation professionnaliste adoptée par les associations d'infirmières n'est pas due aux seules volontés d'une élite d'infirmières, anxieuses de plaire aux médecins et aux directions hospitalières. En ce sens, la réaction des infirmières, laissées pour compte comme à SteJustine, se justifie amplement comme une réaction à une injustice qui leur est faite. Elle se double, en l'occurrence ici, d'une méfiance à l'égard d'un leadership qui leur est étranger par bien des aspects: l'«élite» est canadienne-anglaise, protestante et veut plus de professionnalisme. Vivant, comme elles le font quotidiennement, des réalités très différentes - la présence des religieuses ainsi que le missionnariat qui marque leur engagement hospitalier en sont des composantes notables - elles entendent faire valoir leurs vues aussi. L'association avec la directrice de l'hôpital leur apparaît donc comme une façon relativement commode de faire reconnaître leur travail, tout en obtenant la diplomation nécessaire à un statut qu'elles briguent également. La logique de la protection l'emporte, dans ce cas, sur celle de l'autonomie pratiquée par l'AGMEPQ.

C'est ainsi que les infirmières de ces deux hôpitaux articulent dès les débuts deux stratégies, divergentes par bien des aspects, en vue de

\footnotetext{
56 Un mémoire sera soumis, par les médecins rattachés à la Faculté de médecine de l'Université de Montréal, au Premier ministre du Québec en 1922. Il est prévu notamment: «L'approbation par la Faculté de Médecine du programme d'étude établi dans chaque hôpital catholique de Montréal. La délivrance d'un diplôme de garde-malade par l'Université elle-même.» Et le Docteur Benoît présente en ces termes ce mémoire: «Dès que la faculté de médecine aura reçu votre adhésion, elle se mettra en relation avec vous pour discuter et formuler un projet définitif qui puisse être accepté par tous les hôpitaux catholiques et régler de façon satisfaisante le statut de nos gardes-malades...» AOIIQ, lettre retranscrite dans les procès-verbaux de l'AGMEPQ, 19171925,28 janvier 1922.

57 «Doctors, Patients, and 'Big Nurse': Work and Gender in the Postwar Hospital», in E. C. Lagema-n, ed., Nursing History: New Perspectives, New Possibilities (Philadelphia, Teacher College Press, 1983), 153-180.
} 
faire reconnaître leur métier. Pour les unes, celles de l'Hôpital général de Montréal, il s'agit d'emprunter la voie d'un professionnalisme calqué sur celui des médecins et auquel seule l'association autonome peut mener. Une telle démarche signifie pratiquement l'abandon de références explicites au sexe (don de soi et goût particulier pour le soin) et leur transformation en savoirs infirmiers (propreté, hygiène, etc...). Pour les autres, à Ste-Justine, la communauté des femmes soignantes et soignées justifie une démarche centrée au contraire sur la spécifité de ses besoins. La demande de protection, tant celle de Madame Beaubien envers les médecins que celles d'infirmières envers l'administration hospitalière, traduit sûrement une autre vision du métier d'infirmière. Cette stratégie, qui emprunte à la «sollicitude» féminine ${ }^{58}$ l'ensemble de son arsenal, et à la charité chrétienne son idéologie, aboutit également à la reconnaissance du travail fourni; mais elle n'entre pas en conflit avec les autorités, du moins pas à ses débuts. Ainsi, ces deux stratégies sont dictées aux infirmières pionnières par le milieu auquel elles sont confrontées, mais aussi par les ambitions qu'elles convoitent pour leur profession.

Ces études de cas illustrent bien l'utilisation qu'elles ont pu faire des ressources à leur disposition. Certes, la prise en compte des variables socio-démographiques (lieu de naissance, origine socio-économique, religion, formation académique) s'avère indispensable à l'analyse. Comment, en effet, ne pas voir la différence entre les itinéraires des Canadiennes anglaises et ceux des Canadiennes françaises? Ne faut-il pas l'interpréter comme étant une différence non seulement de culture, mais aussi de classe? À cet égard, les données sur les origines socioprofessionnelles des infirmières nous manquent encore cruellement pour tenter une quelconque approximation. Il n'en demeure pas moins que c'est autour de l'identité féminine que se trouvent aux débuts les principaux enjeux. C'est le degré de transformation du savoir-faire féminin qui détermine l'avenir de la profession. Bien que les stratégies de professionnalisation divergent selon les hôpitaux, les milieux et les classes sociales, les objectifs restent semblables pour toutes les femmes qui embrassent le métier d'infirmière, dans les premières décennies du siècle. Il s'agit de transférer des savoirs féminins en soins infirmiers, au prix d'une plus longue formation, d'un apprentissage peu rémunéré, et d'un rôle complémentaire et subordonné aux médecins. En échange, elles obtiennent un statut professionnel (garde-malade enregistrée) qui leur garantit un emploi et une rémunération sur lesquels elles peuvent exercer un certain contrôle.

Ainsi, les infirmières de l'Hôpital général de Montréal et de SteJustine ont su profiter de l'extension du domaine de la santé, pour y

\footnotetext{
58 In a Different Voice: Psychological Theory and Women's Development (Cambridge, Harvard University Press, 1982).
} 
introduire et faire valoir leurs propres compétences. A partir de là, elles ont su se donner les moyens de négocier un statut d'envergure, sinon de prestige, qui les a mis à l'abri du despotisme administratif, de l'autorité et du contrôle des médecins.

Finalement, c'est d'abord en partant d'une démarche qui s'appuie et valorise des savoirs spécifiquement féminins, que les infirmières ont réussi à inscrire leur rôle dans le secteur de la santé. Toutefois, pour obtenir la reconnaissance puis la rémunération de leurs savoirs, elles ont dû emprunter le cheminement de l'association professionnelle ${ }^{59}$. Suivant en cela l'exemple des autres corps professionnels déjà constitués, en l'occurrence masculins, elles ont compris la nécessité qu'il y avait de se doter d'un organe de représentation officiel apte à négocier la place qui leur revient dans un domaine alors en train de se définir. Ce que cette histoire révèle, en outre, c'est l'influence exercée par des associations professionnelles en matière d'emploi. Tout comme les autres groupes professionnels qui l'ont précédée, l'AGMEPQ, en se réservant le contrôle d'un secteur, s'érige en interlocuteur de ses membres auprès des autorités publiques.

\footnotetext{
59 À ce titre, il est éloquent que les gardes-malades aient été les premières femmes à s'enregistrer, et parmi les six premières professions qui comprennent l'urgence de le faire dans le champ de la santé, quant ce dernier se réorganise sur la base d'une plus grande spécialisation. À cette époque, neuf corporations professionnelles sont formées dont cinq dans le secteur de la santé: homéopathes (1865), dentistes (1869), pharmaciens (1870), médecins vétérinaires (1902) et optométristes (1906); Gilles Dussault, loc. cit., 446.
} 